\title{
PIMS-TS vs. MIS-C: Diagnostic Criteria in COVID-19-Associated Hyperinflammation in Children
}

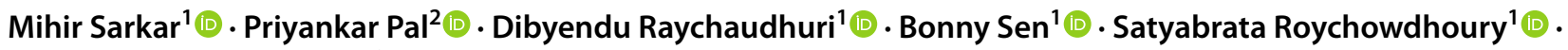 \\ Amitabha Chattopadhyay ${ }^{3}$ (D) Avijit Hazra ${ }^{4}$ (D) Rakesh Mondal $^{5}(\mathbb{C}$
}

Received: 12 May 2021 / Accepted: 16 July 2021 / Published online: 9 August 2021

c) Dr. K C Chaudhuri Foundation 2021

To the Editor: A multicenter study conducted in three COVID care hospitals of Eastern India to evaluate PIMS and MIS-C criteria for identifying COVID-19 associated hyperinflammatory state $[1,2]$ among 1 mo- to 12 -y-old children with hyperinflammation. Those with known hematologic and autoimmune diseases, culture-proven bacterial sepsis, and evidence of concurrent infection were excluded. Inflammatory markers used to diagnose hyperinflammatory states were lymphopenia and/or neutrophilia, elevated C-reactive protein, erythrocyte sedimentation rate, procalcitonin, fibrinogen, D-dimer, serum ferritin, and interleukin-6.

Twenty-one children satisfied both PIMS [3] and MIS-C criteria [4] and 11 children met only PIMS-TS criterion, while none met only MIS-C criterion. Fifty percent had positive nasopharyngeal swabs, $62.5 \%$ documented positive antibody titer, while $25 \%$ had definite contact history.

Fever was noted as the most frequent feature followed by rashes $(78 \%)$, respiratory distress $(53 \%)$, septic shock $(46.8 \%)$, coronary artery abnormalities $(31.3 \%)$, systolic dysfunction (43.8\%), and diastolic dysfunction $(6.3 \%)$. While $72 \%$ patients required intensive care, 2 patients received mechanical ventilation. Radiological lung involvement was seen in $15.6 \%$; and $46.8 \%$ and $62.5 \%$ had elevated

Rakesh Mondal

ivanrakesh2001@gmail.com

1 Department of Pediatrics, Medical College Kolkata, Kolkata, West Bengal, India

2 Department of Pediatrics, Institute of Child Health, Kolkata, West Bengal, India

3 Departmen of Pediatrics, Narayana Superspecialty Hospital, Howrah, West Bengal, India

4 Department of Pharmacology, IPGMER and SSKM Hospital, Kolkata, West Bengal, India

5 Rheumatology Unit, Department of Pediatric Medicine, Medical College Kolkata, Kolkata, West Bengal 700073, India
pro-BNP and D-dimer, respectively. Majority (84.4\%) received immunosuppressants with baseline management, while half of the patients responded to intravenous immunoglobulins and two required biologics. One child succumbed to ARDS and multiorgan failure.

The children satisfying both PIMS-TS and MIS-C criteria had significant association with shock and myocardial dysfunction, higher inotrope requirement, and longer ICU stay than those satisfying only PIMS criterion.

We inferred that employing the only MIS-C criterion had higher probability of missing out the cases of hyperinflammation at early stages. On the other hand, patients who met the only PIMS-TS criterion had reduced intensive care unit stays and less disease severity. Criterion like PIMS-TS can play a decisive role in early identification of COVID19-associated hyperinflammatory state.

\section{Declarations}

Ethical Clearance MC/KOL/IEC/NON-SPON/743/07/20 Dated-24-072020

Conflict of Interest None.

\section{References}

1. Webb BJ, Peltan ID, Jensen P, et al. Clinical criteria for COVID19-associated hyperinflammatory syndrome: a cohort study. Lancet Rheumatol. 2020;2:e754-63.

2. Riphagen S, Gomez X, Gonzalez-Martinez C, Wilkinson N, Theocharis P. Hyperinflammatory shock in children during COVID-19 pandemic. Lancet. 2020;395:1607-8.

3. Royal College of Pediatrics and Child Health. Pediatric multisystem inflammatory syndrome temporally associated with COVID19 (PIMS) - guidance for clinicians. 2020. Available at: https:// www.rcpch.ac.uk/resources/paediatric-multisystem-inflammatorysyndrome-temporally-associated-covid-19-pims-guidance. Accessed on 5 May 2020. 
4. World Health Organization. Multisystem inflammatory syndrome in children and adolescents with COVID-19. 2020. Available at: https:// www.who.int/publications/i/item/multisystem-inflammatorysyndrome-in-children-and-adolescents-with-covid-19. Accessed on 31 May 2021.
Publisher's Note Springer Nature remains neutral with regard to jurisdictional claims in published maps and institutional affiliations. 